\title{
ATRIBUTOS FÍSICOS DO SOLO EM LAVOURA DE CAFEEIRO CONILON SUBMETIDA À SUBSOLAGEM
}

\author{
Joabe Martins de Souza ${ }^{1}$, Robson Bonomo ${ }^{2}$, Fábio Ribeiro Pires ${ }^{3}$, Diego Zancanella Bonomo ${ }^{4}$
}

\begin{abstract}
RESUMO
O preparo do solo modifica os atributos físicos, melhorando o desenvolvimento das culturas agrícolas. O objetivo desse trabalho foi avaliar o efeito residual dos atributos físicos do preparo do solo, realizado no plantio de cafeeiro Conilon (Coffea canephora Pierre). A área do experimento foi composta pelos seguintes talhões: T11, T7 e T3, cultivadas com cafeeiro a 11, 7 e 3 anos, respectivamente, os quais foram submetidos à subsolagem nas linhas de plantio durante sua implantação. Foram retiradas amostras indeformadas na linha (P1) e entrelinha (P2) da cultura nas profundidades de 0,00-0,20, 0,20-0,40, 0,40-0,60, 0,60-0,70 m. O delineamento experimental foi inteiramente ao acaso, em esquema fatorial, com três repetições. Os atributos do solo avaliados foram densidade do solo, porosidade total, macroporosidade e microporosidade e resistência à penetração do solo. A densidade do solo, volume total de poros e macroporosidade apresentaram diferenças significativas entre os pontos até $0,40 \mathrm{~m}$, com maior volume total de poros e macroporos para o ponto P1 e maior densidade do solo para o ponto P2, não diferindo das demais. Houve aumento na microporosidade e diminuição na macroporosidade, em profundidade. Entre os talhões, não foi observada diferença significativa, mostrando que a melhoria da subsolagem persiste ao longo dos anos, nessa condição de cultivo. A resistência à penetração diferiu entre os pontos, ocorrendo um aumento com a profundidade, não diferindo entre os talhões. Foi observada entre densidade e porosidade do solo uma correlação positiva, já para densidade e macroporosidade negativa, concluindo que a subsolagem alterou os atributos do solo, reduziu a resistência à penetração melhorando as condições de cultivo.
\end{abstract}

Palavras-chaves: coffea canephora Pierre, compactação, densidade do solo, porosidade do solo, preparo do solo

\begin{abstract}
SOIL PHYSICAL ATTRIBUTES IN CONILON COFFEE PLANTATION SUBMITTED TO SUBSOILING

Soil prepation modifies its physical attributes, improving crop development. The objective of this study was to evaluate the residual effect of soil preparation on physical attributes, performed in a Conilon coffee plantation (Coffea canephora Pierre). The experimental area consisted of the following plots: T11, T3 and T7 cultivated with coffee for 11, 7 and 3 years, respectively, which were submitted to subsoiling in the rows during their implantation. Undisturbed samples were taken from the row (P1) and between rows (P2) of the culture at depths of 0.00-0.20, 0.20-0.40, 0.40-0.60 and 0.60-0.70 m. The experiment was set up in a completely randomized, factorial design with three replications. The soil attributes evaluated were bulk density, total porosity, macroporosity and microporosity and soil penetration resistance. Soil bulk density, total porosity and macroporosity showed significant differences between the points up to $0.40 \mathrm{~m}$, with higher total porosity and macroporosity for the point $\mathrm{P} 1$ and higher bulk density for the point $\mathrm{P} 2$, not differing from the others. There was an increase in microporosity and decrease in macroporosity at increased depths. Among the plots no significant difference was observed, showing that the improvement of subsoil persists over the years for these culture conditions. Penetration resistance differed between points, increasing with depth, and did not differ between plots. A positive correlation was observed between soil density and porosity, while a negative correlation was shown for density and macroporosity, concluding that subsoiling altered soil properties, reduced penetration resistance, and improved growing conditions of the culture.
\end{abstract}

Keywords: Coffea canephora Pierre, compaction, soil density, soil porosity, soil tillage

\section{Recebido para publicação em 26/10/2013. Aprovado em 28/07/2014.}

1 - Engenheiro Agrônomo, Doutorando em Produção Vegetal UFES/CCA/ Alegre - ES, joabenv@gmail.com

2 - Engenheiro Agrônomo, Professor da UFES/CEUNES/São Mateus - ES, robson.bonomo@gmail.com

3 - Engenheiro Agrônomo, Professor da UFES/CEUNES/São Mateus - ES, pires.fr@gmail.com

4 - Mestre em Agricultura Tropical UFES/CEUNES/São Mateus - ES, diegozancanella@yahoo.com.br 


\section{INTRODUÇÃO}

A agricultura, como importante atividade do setor agropecuário, desempenha função de vital relevância para o desenvolvimento social e econômico do Brasil. Em relação ao Estado do Espírito Santo, esse se destaca principalmente com relação à cultura do cafeeiro Conilon, dentre outras, tendo grande importância econômica e social no norte do Estado. Para manter essa importância, práticas realizadas antes da implantação de uma lavoura cafeeira têm significado especial e, neste contexto, o solo é o principal suporte da produção agrícola.

O preparo do solo está relacionado à sustentabilidade das culturas, pois influência grande parte dos atributos físicos do solo, afetando todo o processo de estabelecimento, desenvolvimento e produção das plantas cultivadas. Estes sistemas devem proporcionar boas condições físicas do solo para que as plantas possam se desenvolver adequadamente (CORTEZ et al., 2011).

Na cafeicultura, o preparo do solo visa melhorar as condições de desenvolvimento do sistema radicular do cafeeiro (LANI et al., 2007), já que as raízes podem ser afetadas por vários atributos físicos do solo, esse preparo é realizado através de implementos como grades e subsoladores.

No Espírito Santo, os solos conhecidos como tabuleiros costeiros ocorrem predominantemente no norte e noroeste do estado (IJSN, 2012). Segundo Moreau et al. (2006), os solos de tabuleiros costeiros além de horizonte coeso, duas outras feições morfológicas são encontradas, fragipã e duripã, sendo estes mais frequentes nas áreas deprimidas da paisagem. As práticas que visam eliminar ou diminuir o horizonte coeso se tornam importantes antes da implantação das culturas nessa região.

As práticas indicadas para implantação da lavoura cafeeira são a aração, gradagem e a subsolagem (LANI et al., 2007), sendo que o preparo do sulco de plantio constitui uma etapa importante desde sistema (SERAFIM et al., 2011). Segundo Vitória et al. (2012), a produtividade vegetal depende de vários fatores, dentre os quais se destacam a densidade e a porosidade do solo.

Assim, o uso de máquinas agrícolas nas várias etapas do processo de produção, em condições inadequadas de umidade, tem sido o principal responsável por acarretar a degradação da estrutura do solo, diminuindo o seu potencial produtivo (ARAÚJO-JÚNIOR et al., 2011; SEVERIANO et al., 2011).
Atributos físicos do solo favoráveis ao crescimento do sistema radicular são necessários para a obtenção e manutenção de elevadas produtividades. Os solos devem possuir suficiente espaço poroso (macro e microporos) para o movimento da água e gases, bem como resistência favorável a penetração e desenvolvimento das raízes (PARENTE; MAIA, 2011), assim a densidade e porosidade total podem mostrar se o solo apresenta problema de compactação e condições adequadas para que a planta possa se desenvolver.

Richart et al. (2005) sugerem algumas práticas com a finalidade de se aliviar a compactação, sendo uma delas a descompactação do solo com auxílio de subsoladores e escarificadores, a adoção dessa e de outras técnicas poderão minimizar os efeitos da compactação do solo, melhorando assim o ambiente para desenvolvimento do sistema radicular das culturas e possibilitando a sustentabilidade da agricultura.

Em plantios de café com compactação superficial, recomenda-se utilizar o subsolador a uma profundidade de 0,2 a $0,3 \mathrm{~m}$, já em áreas com camadas compactadas mais profundas, utiliza-se o implemento em profundidades de 0,5 a $0,7 \mathrm{~m}$ (MATIELLO et al., 2010).

O objetivo desse trabalho foi avaliar o efeito residual dos atributos físicos do preparo do solo, realizado no plantio de cafeeiro Conilon (Coffea canephora Pierre).

\section{MATERIAL E MÉTODOS}

O presente trabalho foi desenvolvido em área com cultivo de cafeeiro Conilon (Coffea canephora Pierre) localizada na rodovia São Mateus - Nova Venécia-ES, latitude $18^{\circ} 43^{\prime} 58,57^{\prime \prime} \mathrm{S}$ e $40^{\circ} 5^{\prime}$ 52,97 " O. O espaçamento empregado é de $3 \times 1 \mathrm{~m}$, sendo utilizado o sistema de poda programada do Conilon, que consiste da renovação do cafezal, por meio de podas drásticas, realizadas a cada quatro anos.

O clima do município de São Mateus é Aw, segundo classificação de Köppen é caracterizado por clima tropical úmido, com inverno seco e chuvas máximas no verão. A precipitação média anual de $1200 \mathrm{~mm}$ concentra-se entre os meses de novembro e janeiro. A temperatura média anual é de $23{ }^{\circ} \mathrm{C}$, e as médias máximas e mínimas são de $29^{\circ} \mathrm{C} \mathrm{e} 18^{\circ} \mathrm{C}$, respectivamente.

O solo da área de estudo foi classificado como Argissolo Amarelo Coeso, textura média em A e argilosa em B (EMBRAPA, 2006).

\section{REVENG}


O delineamento experimental utilizado foi inteiramente ao acaso em esquema fatorial $3 \times 4 \times$ 2, com três repetições, sendo o primeiro fator os 3 talhões com idades diferentes T11 (11 anos), T7 (7 anos) e T3 (3 anos), o segundo fator foram as quatro profundidades amostradas $(0,00-0,20,0,20-0,40$, $0,40-0,60,0,60-0,70 \mathrm{~m})$ e o terceiro fator foram os dois pontos P1 (linha do cafeeiro) e P2 (entrelinha do cafeeiro).

Os três talhões foram submetidos à subsolagem na linha do cafeeiro na época de plantio. Para realização da subsolagem do terreno foi utilizado um trator Valmet, $4 \times$ 4, traçado de $140 \mathrm{cv}$, com subsolador de 4 hastes de 0,50 m (T11 - 11 anos), um trator de esteira Carterpillar D6 com ripper de $0,80 \mathrm{~m}$ ( $\mathrm{T} 7-7$ anos) e um trator de pneu John Deere de $180 \mathrm{cv}$ com ripper de $0,80 \mathrm{~m}$ (T3 - 3 anos).

$\mathrm{O}$ manejo mecanizado da área realizada pelo agricultor ocorre no plantio e a cada 4 anos, com a realização da poda drástica das plantas de $0,20-0,40$ $\mathrm{m}$ de altura do solo, com a utilização de um do trator de pneu Agrale 5754 x4, com 65 cv de potência, munido de um triturador e aplicador de calcário. Ao longo do primeiro ano de implantação da lavoura

Quadro 1. Valores médios de areia grossa, fina, silte e argila em dois pontos, quatro profundidades e três talhões.

\begin{tabular}{|c|c|c|c|c|c|c|}
\hline \multirow[t]{2}{*}{ Talhão } & \multirow[t]{2}{*}{ Ponto } & \multirow[t]{2}{*}{ Camada (m) } & Areia Grossa & $\begin{array}{c}\text { Areia } \\
\text { Fina } \\
\end{array}$ & Argila & Silte \\
\hline & & & \multicolumn{4}{|c|}{$\mathrm{g} \mathrm{kg}^{-1}$} \\
\hline \multirow{8}{*}{ T11 } & \multirow{4}{*}{ P1 } & $0,00-0,20$ & 621,88 & 145,34 & 221,05 & 11,74 \\
\hline & & $0,20-0,40$ & 381,03 & 192,31 & 419,60 & 7,06 \\
\hline & & $0,40-0,60$ & 345,42 & 158,84 & 488,89 & 6,85 \\
\hline & & $0,60-0,70$ & 319,82 & 164,56 & 503,91 & 11,71 \\
\hline & \multirow{4}{*}{ P2 } & $0,00-0,20$ & 619,20 & 156,07 & 218,60 & 6,13 \\
\hline & & $0,20-0,40$ & 441,53 & 181,18 & 362,09 & 15,20 \\
\hline & & $0,40-0,60$ & 309,84 & 152,59 & 518,12 & 19,45 \\
\hline & & $0,60-0,70$ & 306,02 & 152,31 & 534,27 & 7,40 \\
\hline \multirow{8}{*}{$\mathrm{T} 7$} & \multirow{4}{*}{ P1 } & $0,00-0,20$ & 528,27 & 165,12 & 293,06 & 13,55 \\
\hline & & $0,20-0,40$ & 445,27 & 190,65 & 348,75 & 15,33 \\
\hline & & $0,40-0,60$ & 361,52 & 177,17 & 448,19 & 13,13 \\
\hline & & $0,60-0,70$ & 364,46 & 144,92 & 479,11 & 11,51 \\
\hline & \multirow{4}{*}{ P2 } & $0,00-0,20$ & 577,41 & 165,08 & 244,15 & 13,36 \\
\hline & & $0,20-0,40$ & 449,79 & 158,84 & 381,38 & 9,99 \\
\hline & & $0,40-0,60$ & 362,76 & 156,85 & 473,91 & 6,48 \\
\hline & & $0,60-0,70$ & 352,87 & 140,20 & 501,52 & 5,41 \\
\hline \multirow{8}{*}{$\mathrm{T} 3$} & \multirow{4}{*}{ P1 } & $0,00-0,20$ & 507,84 & 178,40 & 299,60 & 14,15 \\
\hline & & $0,20-0,40$ & 421,58 & 183,93 & 374,00 & 20,49 \\
\hline & & $0,40-0,60$ & 373,10 & 158,45 & 458,71 & 9,74 \\
\hline & & $0,60-0,70$ & 355,27 & 140,21 & 489,03 & 15,50 \\
\hline & \multirow{4}{*}{ P2 } & $0,00-0,20$ & 675,03 & 119,04 & 186,06 & 19,87 \\
\hline & & $0,20-0,40$ & 514,98 & 179,32 & 292,30 & 13,41 \\
\hline & & $0,40-0,60$ & 361,69 & 147,71 & 481,22 & 9,38 \\
\hline & & $0,60-0,70$ & 354,26 & 129,47 & 508,37 & 7,90 \\
\hline
\end{tabular}


e no pós-poda, também é realizada a aplicação de adubo foliar e defensivos, com média de cinco entradas utilizando um trator Agrale 4200, 4 x 2, com $30 \mathrm{cv}$ de potência munido de um pulverizador.

Para determinação da densidade do solo e porosidade do solo foram coletadas nos pontos e profundidades anteriormente citadas, amostras com estrutura preservada (indeformadas), através de cilindros com $0,05 \mathrm{~m}$ de diâmetro e $0,03 \mathrm{~m}$ de altura, com o auxílio de um amostrador Uhland. Posteriormente, em laboratório, foi determinada a densidade do solo pelo método do anel volumétrico, a macroporosidade e microporosidade do solo e a porosidade total segundo Embrapa (2011).

Adeterminação da resistência do soloà penetração foi realizada com a utilização de um penetrômetro de impacto modelo Stolf de cone de ponta fina $\left(30^{\circ}\right)$ (STOLF et al., 1983). Foram tomadas as leituras em dois pontos em quatro profundidades, de 0,70 $\mathrm{m}$ em cada talhão, coletando também amostra de solo para determinação da umidade do solo segundo Embrapa (2011). Os dados obtidos no campo na unidade de impactos/decímetro foram transformados em $\mathrm{MPa}$, conforme descrito por Stolf (1991).

A análise textural foi realizada pelo método da pipeta, baseado no princípio da velocidade de queda das partículas, conforme a Lei de Stokes, utilizandose como dispersante químico o hidróxido de sódio (EMBRAPA, 2011) (Quadro 1).

Os resultados obtidos foram submetidos à análise de variância. As médias foram comparadas pelo teste Tukey, em nível de 5\% de probabilidade. Além disso, para verificar relações entre algumas variáveis, foi realizada complementarmente correlação linear de Pearson.

\section{RESULTADOS E DISCUSSÃO}

No Quadro 2, pode-se verificar que a densidade do solo (Ds), o volume total de poros (VTP) e a macroporosidade (MACRO) apresentaram diferença significativa entre os pontos estudados P1(linha do cafeeiro) e P2 (entrelinha do cafeeiro) nas camadas de 0,00-0,20 e 0,20-0,40 m com maior volume total de poros e macroporos para o ponto P1 e uma maior densidade do solo para o ponto $\mathrm{P} 2$, nessas mesmas camadas, não diferindo estatisticamente para as demais camadas estudadas. O volume total de poros apresentou comportamento inverso à densidade do solo (Quadro 2), observando relação entre a estrutura do solo e distribuição de poros. Bavoso et al. (2010) afirmam que a mobilização do solo de textura argilosa através de subsoladores, aeradores e arado altera a estrutura por meio da quebra nas ligações entre agregados e expõe o solo a secagem e umedecimento, contribuindo para os mecanismos que promovem o arranjo de fendas e microfendas, definindo as zonas de fraqueza na estrutura do solo. As quebras desses agregados resultam na alteração na distribuição da porosidade do solo.

Houve aumento da microporosidade em profundidade, com diminuição da macroporosidade proporcionada pelo aumento da densidade do solo, comprovando que no processo de compactação ocorre a transformação de macro em microporos, resultados que se assemelham com Souza et al. (2010) trabalhando com soja em Latossolo Vermelho submetidos a dois sistema de manejos, plantio convencional e plantio direto, porém não apresentou diferença estatística entre os pontos estudados (Quadro 2). Esses mesmo resultados são confirmados também quando se analisa cada talhão e profundidade (Quadro 3).

Os atributos físicos do solo (Quadro 4) não apresentaram diferença significativa entre os talhões com diferentes idades do preparo inicial, confirmando a homogeneidade original do solo entre os talhões da área estudada. Jorge et al. (2012), trabalhando com nove tipos de manejo do solo, em Latossolo Vermelho, constataram que os atributos físicos foram alterados em função do tipo de uso/manejo e época de amostragem, em geral, com efeitos negativos em função do tempo de uso agrícola, diferentemente dos resultados obtidos neste trabalho.

Comparando os pontos dentro de cada talhão, apenas o talhão com 7 anos de idades (T7) apresentou diferença estatística para densidade do solo (Ds), volume total de poros (VTP) e

\section{REVENG}


Quadro 2. Densidade do solo, volume total de poros, microporosidade e macroporosidade, determinados na linha (P1) e na entrelinha (P2), em quatro profundidades de amostragem, na média de três talhões do cafezal Conilon

\begin{tabular}{|c|c|c|}
\hline \multirow{2}{*}{$\begin{array}{l}\text { Profundidade } \\
\text { (m) }\end{array}$} & \multicolumn{2}{|c|}{ Densidade do Solo $\left(\mathrm{g} \mathrm{cm}^{-3}\right)$} \\
\hline & $\mathrm{P} 1$ & $\mathrm{P} 2$ \\
\hline $0,00-0,20$ & $1,40 \mathrm{Cb}$ & $1,60 \mathrm{Aa}$ \\
\hline $0,20-0,40$ & $1,49 \mathrm{BCb}$ & $1,67 \mathrm{Aa}$ \\
\hline $0,40-0,60$ & $1,61 \mathrm{ABa}$ & $1,62 \mathrm{Aa}$ \\
\hline \multirow[t]{2}{*}{$0,60-0,70$} & $1,66 \mathrm{Aa}$ & $1,68 \mathrm{Aa}$ \\
\hline & \multicolumn{2}{|c|}{ Volume Total de Poros $\left(\mathrm{m}^{3} \mathrm{~m}^{-3}\right)$} \\
\hline $0,00-0,20$ & $46,51 \mathrm{Aa}$ & $39,84 \mathrm{Ab}$ \\
\hline $0,20-0,40$ & $46,66 \mathrm{Aa}$ & $39,22 \mathrm{Ab}$ \\
\hline $0,40-0,60$ & $42,43 \mathrm{ABa}$ & $40,54 \mathrm{Aa}$ \\
\hline \multirow[t]{2}{*}{$0,60-0,70$} & $39,64 \mathrm{Ba}$ & 42,98 Аа \\
\hline & \multicolumn{2}{|c|}{ Macroporos $\left(\mathrm{m}^{3} \mathrm{~m}^{-3}\right)$} \\
\hline $0,00-0,20$ & $25,36 \mathrm{Aa}$ & $21,34 \mathrm{Aa}$ \\
\hline $0,20-0,40$ & $23,82 \mathrm{ABa}$ & $16,17 \mathrm{ABb}$ \\
\hline $0,40-0,60$ & $15,73 \mathrm{BCa}$ & $12,84 \mathrm{ABa}$ \\
\hline \multirow[t]{2}{*}{$0,60-0,70$} & $10,27 \mathrm{Ca}$ & $11,38 \mathrm{Ba}$ \\
\hline & \multicolumn{2}{|c|}{ Microporos $\left(\mathrm{m}^{3} \mathrm{~m}^{-3}\right)$} \\
\hline $0,00-0,20$ & $21,15 \mathrm{Ba}$ & $18,50 \mathrm{Ca}$ \\
\hline $0,20-0,40$ & $22,84 \mathrm{ABa}$ & $23,05 \mathrm{BCa}$ \\
\hline $0,40-0,60$ & $26,70 \mathrm{ABa}$ & $27,69 \mathrm{ABa}$ \\
\hline $0,60-0,70$ & $29,37 \mathrm{Aa}$ & $31,60 \mathrm{Aa}$ \\
\hline
\end{tabular}

Médias seguidas pelas mesmas letras maiúsculas na coluna e minúsculas na linha, não diferem estatisticamente entre si pelo teste de Tukey a 5\% de probabilidade. Coeficiente de variação (CV\%) da Ds, VTP, MACRO e MICRO, 4,35 $\%, 6,06 \%, 26,33 \%$ e $14,17 \%$, respectivamente.

macroporosidade (MACRO), mostrando que essas propriedades foram inferiores estatisticamente a entrelinha do cafezal. Sendo que o ponto P1, onde ocorreu a subsolagem, apresentou maiores médias apesar de não significativo para essas propriedades nos demais talhões (Quadro4). Nacif et al. (2008), trabalhando com Latossolo Amarelo em região de Tabuleiros Costeiros, onde apresentam uma camada coesa superficial, encontram resultados semelhantes, avaliando as consequências da subsolagem no atributos físicos do solo.

O volume total de poros apresentou uma correlação inversa entre os pontos para microporosidade, sendo que em P1 apresentouse negativa $(r=-0,49)$ e positiva $(r=0,47)$ para $\mathrm{P} 2$. Esse resultado mostra que a volume total de poros no $\mathrm{P} 2$ está relacionado à microporosidade, efeito esse da compactação do solo nesse ponto, não apresentando correlação significativa para macroporosidade do solo (Figura 1). O P1 


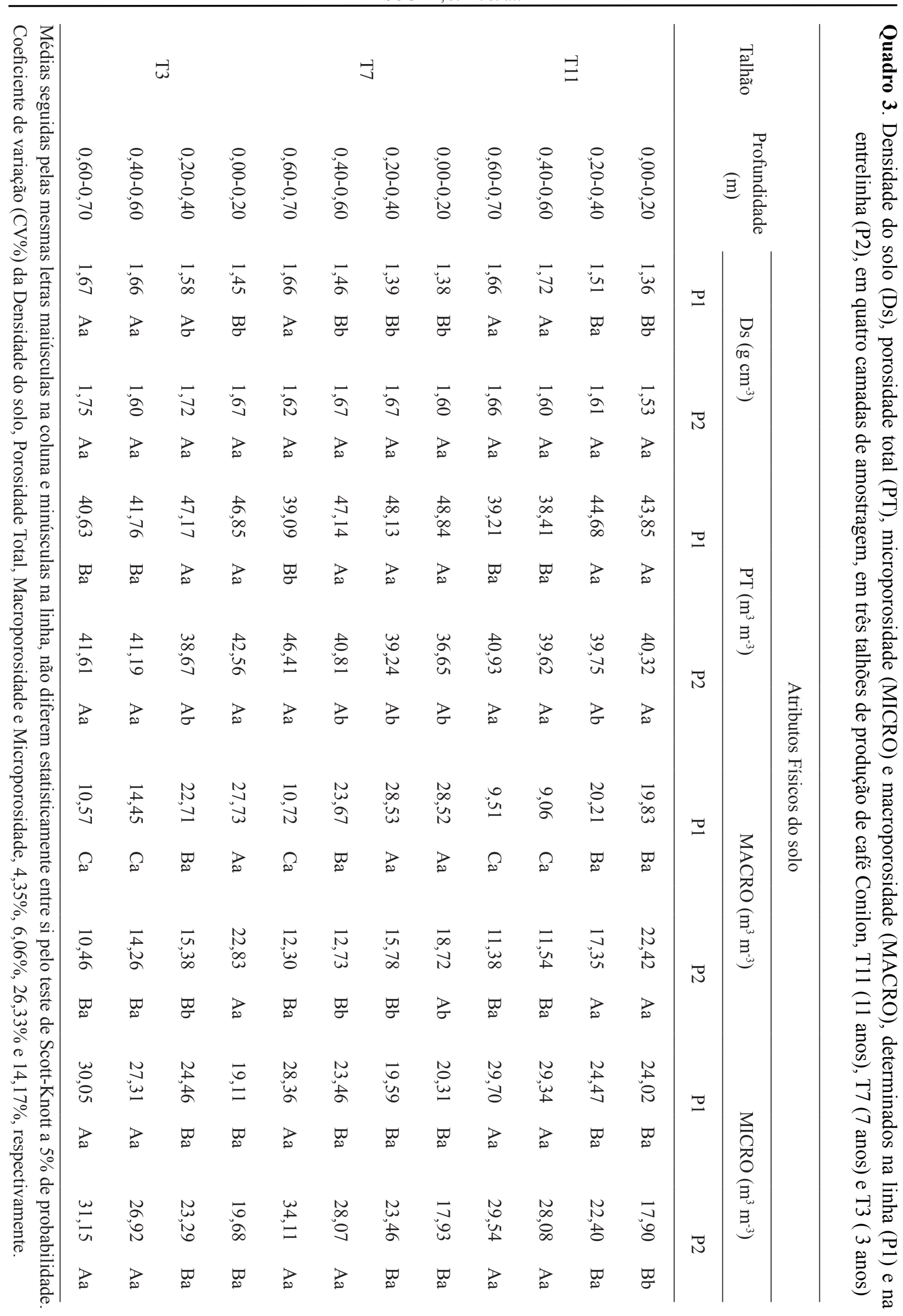


Quadro 4. Densidade do solo (Ds), volume total de poros (VTP), microporosidade (MICRO) e macroporosidade (MACRO) determinados na linha (P1) e na entrelinha (P2), em três talhões do cafezal Conilon, T11 (11 anos), T7 (7 anos) e T3 ( 3 anos)

\begin{tabular}{|c|c|c|}
\hline \multirow{2}{*}{ Talhão } & \multicolumn{2}{|c|}{ Densidade do Solo $\left(\mathrm{g} \mathrm{cm}^{3}\right)$} \\
\hline & P1 & P2 \\
\hline T11 & $1,56 \mathrm{Aa}$ & $1,60 \mathrm{Aa}$ \\
\hline $\mathrm{T} 7$ & $1,47 \mathrm{Ab}$ & $1,64 \mathrm{Aa}$ \\
\hline \multirow[t]{2}{*}{$\mathrm{T} 3$} & $1,59 \mathrm{Aa}$ & $1,68 \mathrm{Aa}$ \\
\hline & \multicolumn{2}{|c|}{ Volume Total de Poros $\left(\mathrm{m}^{3} \mathrm{~m}^{-3}\right)$} \\
\hline T11 & 41,54 Аa & $40,15 \mathrm{Aa}$ \\
\hline $\mathrm{T} 7$ & 45,80 Аа & $40,78 \mathrm{Ab}$ \\
\hline \multirow[t]{2}{*}{$\mathrm{T} 3$} & 44,10 Аа & $41,00 \mathrm{Aa}$ \\
\hline & \multicolumn{2}{|c|}{ Macroporos $\left(\mathrm{m}^{3} \mathrm{~m}^{-3}\right)$} \\
\hline T11 & $14,65 \mathrm{Aa}$ & $15,67 \mathrm{Aa}$ \\
\hline $\mathrm{T} 7$ & 22,86 Аa & $14,88 \mathrm{Ab}$ \\
\hline \multirow[t]{2}{*}{$\mathrm{T} 3$} & 18,86 Аа & $15,74 \mathrm{Aa}$ \\
\hline & \multicolumn{2}{|c|}{ Microporos $\left(\mathrm{m}^{3} \mathrm{~m}^{-3}\right)$} \\
\hline T11 & $26,88 \mathrm{Aa}$ & $24,48 \mathrm{Aa}$ \\
\hline $\mathrm{T} 7$ & $22,93 \mathrm{Aa}$ & $25,89 \mathrm{Aa}$ \\
\hline T3 & 25,23 Aa & $25,26 \mathrm{Aa}$ \\
\hline
\end{tabular}

Médias seguidas pelas mesmas letras maiúsculas na coluna e minúsculas na linha, não diferem estatisticamente entre si pelo teste de Tukey a $5 \%$ de probabilidade. Coeficiente de variação (CV\%) da Ds, VTP, MACRO e MICRO, 4,35 $\%, 6,06 \%, 26,33 \%$ e $14,17 \%$, respectivamente.

apresentou alta correlação $(\mathrm{r}=0,87)$ com a macroporosidade do solo (Figura 1), mostrando o efeito da subsolagem nesse ponto. Medeiros et al. (2013) verificaram que a subsolagem realizada nas entrelinhas das plantas do pomar de citros modificou as propriedades físicas e hídricas do solo, refletindo em aumento da macroporosidade e condutividade hidráulica não saturada e redução da densidade do solo, do grau de compactação e da resistência à penetração.

Parente e Maia (2011) relatam tendência de que com o aumento da densidade do solo, ocorra diminuição da porosidade total, macroporosidade, condutividade hidráulica, absorção iônica, assim como o conseqüente aumento da microporosidade e da resistência mecânica à penetração do solo.

A resistência do solo a penetração apresentou (Quadro 5) valores menores em P1 comparado ao P2 para todas as profundidades estudadas diferindo significativamente do mesmo, ocorrendo um aumento da RP à medida que aumenta a profundidade do solo tanto para $\mathrm{P} 1 \mathrm{e} \mathrm{P} 2$. A umidade do solo, no momento dos testes de RP, não diferiu estatisticamente para os dois pontos com relação às profundidades estudadas, com maiores médias para as camadas de 0,40-0,70 m no P1 e P2. Entre os pontos, apenas na camada de 0,40-0,60 m ocorreu diferença estatística. Pode-se observar também que tanto a resistência mecânica a penetração como a umidade do solo apresentaram diferença estatística para profundidades. 

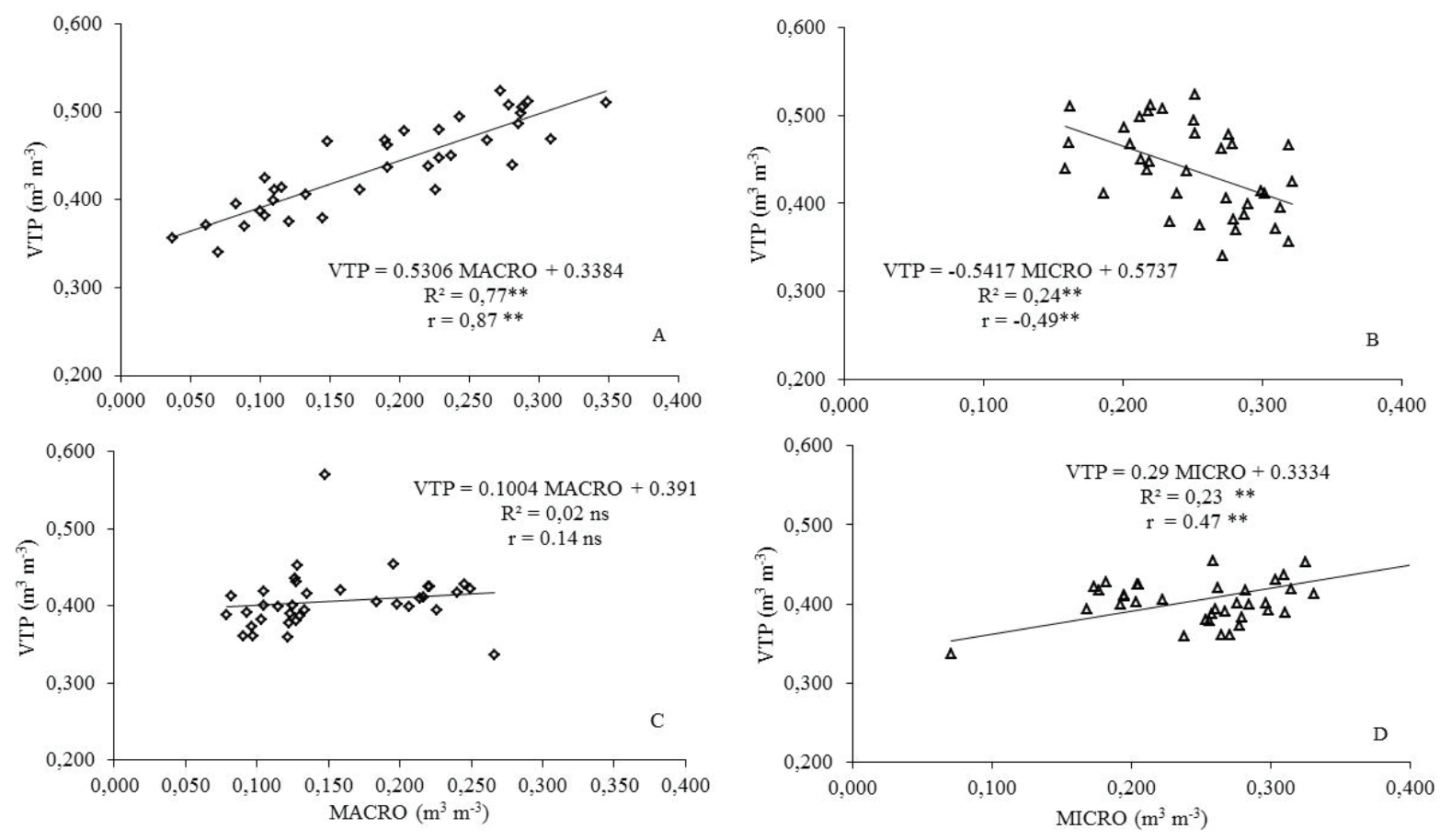

Figura 1. Correlação de Pearson e regressão linear entre volume total de poros (VTP) e macroporosidade (MACRO) e microporosidade (MICRO) no P1(A e B) e P2 (C e D) ( $\mathrm{r} * * *$ : Significativo a 1 ou $5 \%$ respectivamente pelo t, $\mathrm{R}^{2 * *}$ : Significativo a $1 \%$, respectivamente pelo teste de F).

Analisando a resistência mecânica a penetração nos talhões T7 e T3, o P1 apresentou menor resistência em relação ao ponto $\mathrm{P} 2$, sendo que a umidade do solo diferiu entre os pontos apenas no talhão T11 (Quadro 6). Já para o P2 a RP e a umidade do solo não apresentaram diferenças estatísticas entre os talhões, exceto para T11 com relação a RP. Os valores do coeficiente de variação $(\mathrm{CV})$ mostraram pouca variabilidade dos dados obtidos, Mion et al. (2012) encontraram, trabalhando com um Argiloso amarelo RP nas camadas de 0,00-0,10 e $0,10-0,20 \mathrm{~m}$, um CV médio ( $48 \%$ e $58 \%$ ). A RP na camada de 0,20-0,30 m apresentou um CV alto (63\%), conforme limites propostos por Warrick e Nielsen (1980), para classificação de atributos do solo, resultado que diferem desse trabalho com $15 \%$ de coeficiente de variação.

Santos et al. (2012) encontraram valores de CV de 51,68\% para variável resistência do solo à penetração das raízes, considerada de média variabilidade. Guedes Filho et al. (2010) encontraram valores de CV de 44,92\% para a RP em Latossolo Vermelho, avaliada na profundidade de $0,00-0,20 \mathrm{~m}$, mostrando assim a baixa variabilidade dos dados nesse trabalho.

Independente do talhão, a camada de 0,00 a 0,20 $\mathrm{m}$ apresentou valores estatisticamente inferior à 2,0 MPa (Quadro 7), considerado como fator limitante para o desenvolvimento radicular, segundo Senra et al. (2007). Para camada de 0,20-0,40 $\mathrm{m}$ os valores de RP foram menores que 2,5 $\mathrm{MPa}$, exceto para o $\mathrm{T} 7$, valor esse considerado como o máximo para desenvolvimento das culturas segundo Unger e Kaspar (1994), já Hamza e Anderson (2005) relatam valores de 2,5 a 3,0 MPa.

Esses resultados demonstram que a subsolagem foi efetiva até a profundidade de $0,40 \mathrm{~m}$, proporcionando uma melhoria para o desenvolvimento radicular, já que, segundo Partelli et al. (2006), a maior concentração de raízes (comprimento e área superficial por volume de solo) da espécie propagada via seminal ou estaquia encontra-se na camada superficial, com aproximadamente $50 \%$ na camada de $0,00-0,10$ m e mais de $65 \%$ na camada de $0,00-0,20$ $\mathrm{m}$ de solo, ficando menos de $30 \%$ para as camada de 0,20-0,60 m. 
Quadro 5. Resistência do solo à penetração $(\mathrm{RP})$ e umidade do solo, determinadas na linha (P1) e na entrelinha (P2), em quatro profundidades de amostragem, na média de três talhões de cafezl Conilon

\begin{tabular}{|c|c|c|}
\hline \multirow{2}{*}{$\begin{array}{l}\text { Profundidade } \\
\text { (m) }\end{array}$} & \multicolumn{2}{|c|}{$\mathrm{RP}(\mathrm{MPa})$} \\
\hline & $\mathrm{P} 1$ & $\mathrm{P} 2$ \\
\hline $0,00-0,20$ & $1,54 \mathrm{Cb}$ & $3,77 \mathrm{Ca}$ \\
\hline $0,20-0,40$ & $2,60 \mathrm{BCb}$ & $8,91 \mathrm{Ba}$ \\
\hline $0,40-0,60$ & $4,42 \mathrm{Bb}$ & $10,88 \mathrm{ABa}$ \\
\hline \multirow[t]{2}{*}{$0,60-0,70$} & $8,07 \mathrm{Ab}$ & 13,09 Аа \\
\hline & \multicolumn{2}{|c|}{ Umidade do solo $\left(\mathrm{cm}^{3} \mathrm{~cm}^{-3}\right)$} \\
\hline $0,00-0,20$ & $0,165 \mathrm{Ca}$ & $0,176 \mathrm{Ca}$ \\
\hline $0,20-0,40$ & $0,264 \mathrm{Ba}$ & $0,247 \mathrm{Ba}$ \\
\hline $0,40-0,60$ & $0,286 \mathrm{ABa}$ & $0,259 \mathrm{ABb}$ \\
\hline $0,60-0,70$ & 0,293 Аа & 0,280 Аа \\
\hline
\end{tabular}

Médias seguidas pelas mesmas letras maiúsculas na coluna e minúsculas na linha, não diferem estatisticamente entre si pelo teste de Tukey a 5\% de probabilidade. Coeficiente de variação (CV\%) da RP e umidade do solo 15,41\% e 5,08 $\%$, respectivamente.

Quadro 6. Resistência do solo à penetração $(\mathrm{RP})$ e umidade do solo, determinadas na linha $(\mathrm{P} 1)$ e na entrelinha (P2), em três talhões de cafezal Conilon, T11 (11 anos), T7 (7 anos) e T3 ( 3 anos)

\begin{tabular}{|c|c|c|}
\hline \multirow{2}{*}{ Talhão } & \multicolumn{2}{|c|}{$\mathrm{RP}(\mathrm{MPa})$} \\
\hline & $\mathrm{P} 1$ & $\mathrm{P} 2$ \\
\hline T11 & $3,05 \mathrm{Ab}$ & $7,04 \mathrm{Ba}$ \\
\hline $\mathrm{T} 7$ & $4,90 \mathrm{Ab}$ & $10,29 \mathrm{Aa}$ \\
\hline \multirow[t]{2}{*}{$\mathrm{T} 3$} & $4,51 \mathrm{Ab}$ & $10,15 \mathrm{Aa}$ \\
\hline & \multicolumn{2}{|c|}{ Umidade do solo $\left(\mathrm{cm}^{3} \mathrm{~cm}^{-3}\right)$} \\
\hline T11 & $0,253 \mathrm{Aa}$ & $0,232 \mathrm{Ab}$ \\
\hline $\mathrm{T} 7$ & 0,245 Аа & 0,244 Аа \\
\hline T3 & $0,257 \mathrm{Aa}$ & $0,243 \quad \mathrm{Aa}$ \\
\hline
\end{tabular}

Médias seguidas pelas mesmas letras maiúsculas na coluna e minúsculas na linha, não diferem estatisticamente entre si, pelo teste de Tukey, a 5\% de probabilidade. Coeficiente de variação (CV\%) da RP e umidade do solo 15,41\% e $5,08 \%$, respectivamente.

Silva e Martins (2010), trabalhando com atributos físicos do solo de cafezal Conilon, também verificaram que as raízes se concentram na faixa mais superficial do solo, diminuindo em profundidade à medida que se aproximam da profundidade de 0,40 m. Embora os trabalhos anteriores mostrem uma tendência de concentração de raízes nas camadas mais superficiais do solo, um aprofundamento desse sistema radicular irá possibilitar um aumento da capacidade de utilização 
da água armazenada no solo, reduzindo os riscos do estresse hídrico em razão da interrupção das irrigações, ou mesmo de um maior intervalo entre chuvas.

Foi observada correlação positiva $(\mathrm{r}=0,45)$ entre RP e Ds e também $(r=0,49)$ entre RP e MICRO para o P1 (Figura 2). Neste mesmo ponto, observou-se uma correlação negativa $(\mathrm{r}=-0,52)$ com MACRO. Utilizando-se valores limites de MACRO de $0,09 \mathrm{~m}^{3} \mathrm{~m}^{-3}$ na equação de regressão, obtém-se valor de RP igual a 10,86 Mpa. Esse valor representa uma Ds de $1,70 \mathrm{~g} \mathrm{~cm}^{-3}$, sendo que valores superiores a esse dificultaria a aeração das plantas para o P1, limite esse superior ao proposto por Matiello et al. (2002). Os autores supracitados afirmam que, devido ao cafeeiro Conilon apresentar raízes mais vigorosas, a densidade do solo crítica para o desenvolvimento radicular está em torno de $1,45 \mathrm{~g} \mathrm{~cm}^{3}$. Segundo os autores, as raízes de cafeeiro Conilon só não são capazes de romper solos com densidade acima de $1,80 \mathrm{~g} \mathrm{~cm}^{3}$.

Uma correlação positiva foi encontrada com a MICRO $(r=0,69)$ devido à compactação do solo que altera a distribuição das partículas, resultando no seu acondicionamento, ou seja, aproximando-as e incrementando a presença de poros de tamanho menor (Figura 1).

O VTP apresentou correlação inversa entre o P1 e P2, sendo negativa $(\mathrm{r}=-0,41)$, e positiva $(r=0,40)$, respectivamente (Figuras 2 e 3$)$. Esse resultado de $\mathrm{P} 2$ pode ser explicado, já que o VTP em P2 apresenta uma correlação positiva com a microporosidade (Figura 5), fazendo com que o aumento de VTP nesse ponto aumente a RP, mostrando que as práticas de manejo da cultura na entrelinhas do cafezal, associado ao tráfego de máquinas e implementos por bitolas diferenciada, proporciona compactação da superfície numa maior dimensão de área.

Quadro 7. Resistência do solo à penetração $(\mathrm{RP})$ e umidade do solo determinados na linha $(\mathrm{P} 1)$ e na entrelinha (P2), em quatro profundidades de amostragem, em três talhões de cafezal Conilon, T11 (11 anos), T7 (7 anos) e T3 ( 3 anos)

\begin{tabular}{ccccll}
\hline \multirow{2}{*}{ Talhão } & $\begin{array}{c}\text { Profundidade } \\
(\mathrm{m})\end{array}$ & \multicolumn{2}{c}{$\mathrm{RP}(\mathrm{Mpa})$} & \multicolumn{1}{c}{ Umidade $\left(\mathrm{m}^{3} \mathrm{~m}^{-3}\right)$} \\
\cline { 2 - 6 } & $0,00-0,20$ & $1,34 \mathrm{Da}$ & $3,05 \mathrm{Fa}$ & $0,158 \mathrm{Da}$ & $0,165 \mathrm{Da}$ \\
\multirow{2}{*}{ T11 } & $0,20-0,40$ & $2,03 \mathrm{CDb}$ & $7,73 \mathrm{DEa}$ & $0,264 \mathrm{BCa}$ & $0,234 \mathrm{Cb}$ \\
& $0,40-0,60$ & $3,97 \mathrm{BCDb}$ & $8,41 \mathrm{CDa}$ & $0,301 \mathrm{Aa}$ & $0,251 \mathrm{ABCb}$ \\
& $0,60-0,70$ & $4,88 \mathrm{BCb}$ & $8,98 \mathrm{CDa}$ & $0,288 \mathrm{ABa}$ & $0,278 \mathrm{ABa}$ \\
& $0,00-0,20$ & $1,80 \mathrm{Da}$ & $3,51 \mathrm{Fa}$ & $0,168 \mathrm{Da}$ & $0,181 \mathrm{Da}$ \\
& $0,20-0,40$ & $3,74 \mathrm{BCDb}$ & $9,78 \mathrm{CDa}$ & $0,251 \mathrm{Ca}$ & $0,249 \mathrm{BCa}$ \\
& $0,40-0,60$ & $4,19 \mathrm{BCDb}$ & $12,97 \mathrm{ABa}$ & $0,264 \mathrm{BCa}$ & $0,271 \mathrm{ABa}$ \\
& $0,60-0,70$ & $9,89 \mathrm{Ab}$ & $14,91 \mathrm{Aa}$ & $0,297 \mathrm{ABa}$ & $0,276 \mathrm{ABb}$ \\
& $0,00-0,20$ & $1,45 \mathrm{Db}$ & $4,77 \mathrm{EFa}$ & $0,168 \mathrm{Da}$ & $0,180 \mathrm{Da}$ \\
& $0,20-0,40$ & $2,03 \mathrm{CDb}$ & $9,21 \mathrm{CDa}$ & $0,275 \mathrm{ABCa}$ & $0,254 \mathrm{ABCb}$ \\
& $0,40-0,60$ & $5,11 \mathrm{Bb}$ & $11,26 \mathrm{BCa}$ & $0,291 \mathrm{ABa}$ & $0,254 \mathrm{ABCb}$ \\
& $0,60-0,70$ & $9,44 \mathrm{Ab}$ & $15,37 \mathrm{Aa}$ & $0,293 \mathrm{ABa}$ & $0,286 \mathrm{Aa}$ \\
\hline
\end{tabular}

Médias seguidas pelas mesmas letras maiúsculas na linha e minúsculas na coluna, não diferem estatisticamente, entre si, pelo teste de Tukey, a 5\% de probabilidade. Coeficiente de variação (CV\%) da RP e umidade do solo $15,41 \%$ e $5,08 \%$, respectivamente. 

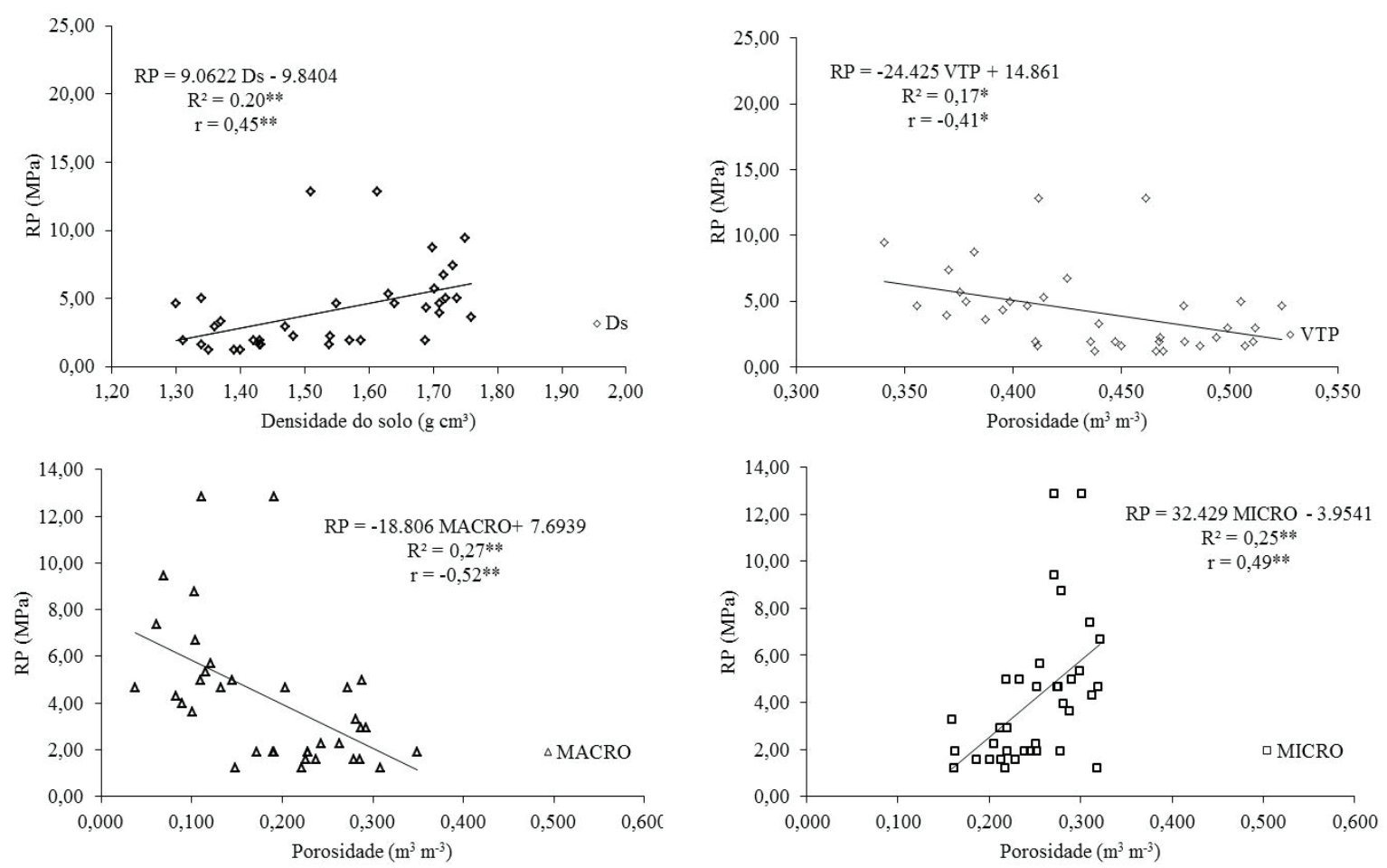

Figura 2. Correlação de Pearson entre resistência à penetração do solo (RP) com atributos físicos do solo no P1. ( $\mathrm{r} * * *$ : Significativo a 1 ou $5 \%$ respectivamente pelo teste $\mathrm{t}, \mathrm{R}^{2} * * *$ : Significativo a 1 ou $5 \%$, respectivamente, pelo teste $\mathrm{F}$ ).
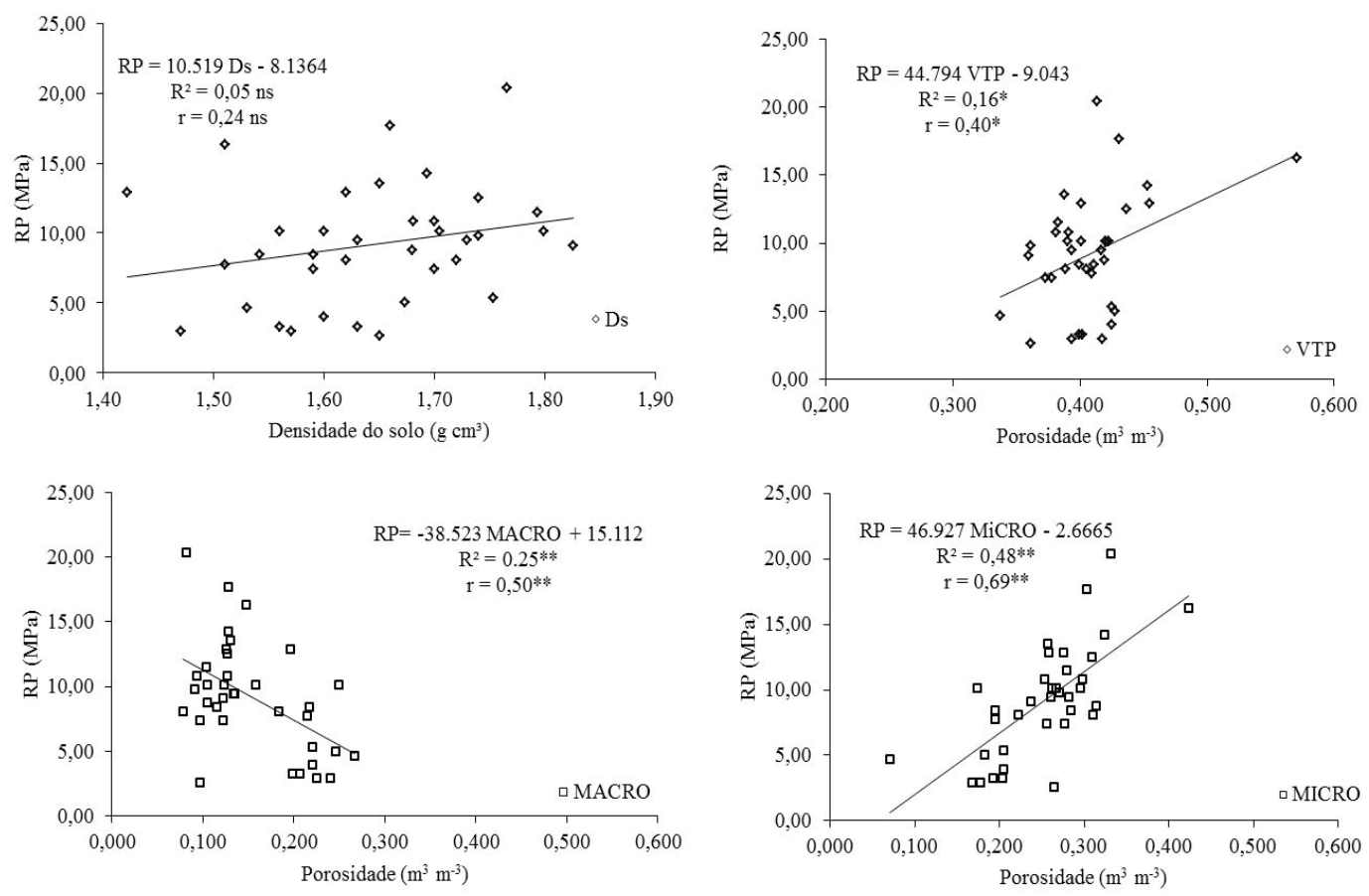

Figura 3. Correlação de Pearson entre resistência à penetração do solo (RP) com atributos físicos do solo no P2. ( $\mathrm{r} * * *$ : Significativo a 1 ou $5 \%$ respectivamente pelo teste $\mathrm{t}, \mathrm{R}^{2 * * *}$ : Significativo a 1 ou $5 \%$, respectivamente, pelo teste $\mathrm{F}$ ). 


\section{CONCLUSÕES}

- A subsolagem alterou os atributos do solo, diminuindo densidade do solo e aumentando o volume total de poros, não ocorrendo efeito ao longo do tempo na linha de plantio do cafezal;

- A subsolagem efetiva foi até a camada de 0,40 $\mathrm{m}$, com seus efeitos benéficos mantidos ao longo de vários anos;

- A subsolagem reduziu a resistência à penetração na linha do cafeeiro, que correlaciou-se positivamente com densidade e microporosidade e negativamente com macroporosidade e volume total de poros.

\section{REFERÊNCIAS}

ARAÚJO-JÚNIOR, C.F.; DIAS JÚNIOR, M.S.; GUIMARÃES, P.T.G. \& ALCÂNTARA, E.N. Capacidade de suporte de carga e umidade crítica de um Latossolo induzida por diferentes manejos. Revista Brasileira de Ciência do Solo, Viçosa, v.35, n.1, p.115-131, 2011.

BAVOSO, M.A.; GIAROLA, N.F.B.; TORMENA, C.A.; PAULETTI, V. Preparo do solo em áreas de produção de grãos, silagem e pastejo: efeito na resistência tênsil e friabilidade de agregados. Revista Brasileira de Ciência do Solo, Viçosa, v.34, n.1, p.235-244, 2010.

BERGAMIN, A.C.; VITORINO, A.C.T.; FRANCHINI, J.C.; SOUZA, C.M.A. \& SOUZA, F.R. Compactação em um Latossolo Vermelho Distroférrico e suas relações com o crescimento radicular do milho. Revista Brasileira de Ciência do Solo, Viçosa, v.34, n.3, p.681-691, 2010.

CORTEZ, J.W.; SILVA ALVES, A.L.; MOURA, M.R.D.; OLSZEVSKI, N.; NAGAHAMA, H.J. Atributos físicos do argissolo amarelo do semiárido nordestino sob sistemas de preparo. Revista Brasileira de Ciência do Solo, Viçosa, v.35, n.4, p.1207-1216, 2011.

EMPRAPA. Sistema brasileiro de classificação dos solos. 2.ed. Rio de Janeiro: Embrapa CNPS, 2006. 306p.

EMBRAPA. Manual de métodos de análise de solo. 2.ed. Rio de Janeiro, 2011. 230p.

GUEDES FILHO, O.; VIEIRA, S.R.; CHIBA, M.K.; NAGUMO, C.H.; DECHEN, C.F. Spatial and temporal variability of crop yield and some rhodic hapludox properties under no-tillage. Revista Brasileira de Ciência do Solo, Viçosa, v.34, n.1, p.1-14, 2010.

HAMZA, M.A.; ANDERSON, W.K. soil compaction in cropping systems. A review of the nature, causes and possible solutions. Soil \& Tillage Research, Amsterdam v.82, p.121-145, 2005.

IJSN. Mapeamento geomorfológico do estado do Espírito Santo. Vitória, ES, 2012. 19f.: il. (Nota técnica, 28).

JORGE, R.F.; ALMEIDA, C.X.; BORGES, E.N.; PASSOS, R.R. Distribuição de poros e densidade de Latossolos submetidos a diferentes sistemas de uso e manejo. Bioscience Journal, Uberlândia, v.28, Supplement 1, p.159-169, 2012.

LANI, J.A.; BRAGANÇA, S.M.; PREZOTTI, L.C.; MARTINS, A.G.; DADALTO, G.G.. IN: FERRÃO, R.G.; FONSECA, A.F.A.; BRAGANÇA, S.M.; FERRÃO, M.A.G.; DE MUNNER, L.H. (Eds.). Preparo, manejo e conservação do solo. 1.ed. Café Conilon: Vitória, 2007, p.281-293.

MAGALHÃES, W.A.; CREMON, C.; MAPELI, N.C.; SILVA, W.M.; CARVALHO, J.M.; MOTA, M.S. Determinação da resistência do solo a penetração sob diferentes sistemas de cultivo em um Latossolo sob Bioma Pantanal. Agrarian, Dourados, v.2, n.6, p.21-32, 2009.

MATIELLO, J.B.; SANTINATO, R.; GARCIA, A.W.R.; ALMEIDA, S.R.A.; FERNANDES, D.R. Cultura do Café no Brasil: Manual de Recomendações. Rio de Janeiro e Varginha: Fundação Procafé, 2010. 542p.

MATIELLO, J.B.; SANTINATO, R.; GARCIA, A.W.R.; ALMEIDA, S.R.; FERNADES, D.R. Cultura do café no Brasil: novo manual de recomendações. Rio de Janeiro: MAPA/ PROCAFE, 2002. 387p.

MEDEIROS, J.C.; COUTINHO, F.G; MAFRA, Á.L.; DALLA R.J.; WON, Y.S. Deep subsoiling of a subsurface-compacted typical hapludult under citrus orchard. Revista Brasileira de Ciência do Solo, Viçosa, v.37, n.4, p.911-919, 2013.

MION, R.L.; NASCIMENTO, E.M.S.; LIMA SALES, F.A.; SILVA, S.F.; DUARTE, J.M.L.; SOUSA, B.M. Variabilidade espacial da porosidade total, umidade e resistência do solo à penetração 
de um Argissolo Amarelo. Semina: Ciências Agrárias, Londrina, v.33, n.6, p.2057-2066, 2012.

MOREAU, A.M.S.S.; COSTA, L.M.; KER, J.C.; GOMES, F.H. Gênese de horizonte coeso, fragipã e duripã em solos do Tabuleiro Costeiro do sul da Bahia. Revista Brasileira de Ciência do Solo, Viçosa, v.30, n.6, p.1021-1030, 2006.

NACIF, P.G.S.; REZENDE, J.O.; FONTES, L.E.F.; COSTA, L.M.; COSTA, O.V. Efeitos da subsolagem em propriedades físico-hídricas de um Latossolo amarelo distrocoeso do Estado da Bahia. Magistra, Cruz das Almas-BA, v.20, n.2, p.186192, 2008.

PARENTE, H.N.; MAIA, M.O. Impacto do pastejo sobre a compactação dos solos a com ênfase no semiárido. Revista Trópica, Chapadinha, v.5, n.3, p.3-15, 2011.

PARTELLI, F.L., VIEIRA, H.D.; SANTIAGO; A.R.; BARROSO, D.G. Produção e desenvolvimento radicular de plantas de café 'Conilon' propagadas por sementes e por estacas. Pesquisa Agropecuária Brasileira, Brasília, v.41, n.6, p.949-954, 2006.

RIBEIRO, M.A.V.; NOVAIS, R.N.; FAQUIN, V.; FERREIRA, M.M.; FURTINI NETO, A.E.; LIMA, J.M.; VILLANI, E.M.A. Resposta da soja e do eucalipto ao aumento da densidade do solo e a doses de fósforo. Revista Brasileira de Ciência do Solo, Viçosa, v.34, n.4, p.1157-1164, 2010.

RICHART, A.; FILHA, J.T.; BRITO, O.R.; LLANILLO, R.F.; FERREIRA, R. Compactação do solo: causas e efeitos. Semina: Ciências Agrárias, Londrina, v. 26, n.3, p.321-344, 2005.

SANTOS, K.S.; MONTENEGRO, A.A.A.; ALMEIDA, B.G.; MONTENEGRO, S.M. G.L.; ANDRADE, T.S.; FONTES JÚNIOR, R.V.P. Variabilidade espacial de atributos físicos em solos de vale aluvial no semiárido de Pernambuco. Revista Brasileira de Engenharia Agrícola e Ambiental, Viçosa, v.16, n.8, p.828-835, 2012.

SENRA, A.F.; LOUZADA, R.O.; VITORINO, A.C.T.; SOUZA, C.M.A.; VICTOR,

D.M. Resistência à penetração em "Latossolo Vermelho" sob diferentes sistemas de uso e manejo do solo. Revista Ciências Técnicas Agropecuárias, La Havana, v.16, n.1, 2007.

SERAFIM ,M.E.; OLIVEIRA, G.C.; OLIVEIRA,
A.S.; LIMA,J.M.; GUIMARÃES, P.T.; COSTA, J.C. Sistema conservacionista e de manejo intensivo do solo no cultivo de cafeeiros na região do alto São Francisco, MG: um estudo de caso. Bioscience Journal, Uberlândia, v.27, n.6, p.964977, 2011.

SEVERIANO, E.C.; OLIVEIRA， G.C.; DIAS JÚNIOR, M.S.; COSTA, K.A.P.; BENITES, V.M.; FERREIRA FILHO, S.M. Structural changes in Latosols of the Cerrado region: ii - soil compressive behavior and modeling of additional compaction. Revista Brasileira de Ciência do Solo, Viçosa, v.35, n.3, p.783-791, 2011.

SILVA, V.L.B.; MARTINS, P.F.S. Propriedades físicas do solo e sistema radicular do cafeeiro, variedade Conilon, sob diferentes espaçamentos. Revista de Ciências Agrárias, Belém, v.53, n.1, p.96-101, 2010.

SOUZA，F.R. S.; JUNIOR, E.J.R.J.; FIETZ, C.R.; BERGAMIN, A.C.; VENTUROSO, L.R.; ROSA, Y.B.C.J. Atributos físicos e desempenho agronômico da cultura da soja em um latossolo vermelho distroférrico submetido a dois sistemas de manejos. Ciência e Agrotecnologia, Lavras, v.34, n.6, p.1357-1364, 2010.

SCHIAVO, J.A.;COLODRO, G.Agregação e resistência à penetração de um Latossolo Vermelho sob sistema de integração lavourapecuária. Bragantia, Campinas, v.71, n.3, p.406412, 2012.

STOLF, R. Teoria e teste experimental de fórmulas de transformação dos dados de penetrômetro de impacto em resistência do solo. Revista Brasileira de Ciência do Solo, Viçosa, v.15, n.2, p.229-35, 1991.

STOLF, R.; FERNANDES, J.; FURLANI NETO, V.L. Penetrômetro de impacto IAA/PlanalsucarSTOLF (Recomendações para seu uso). STAB: Açúcar, Álcool e Subprodutos, Piracicaba, v.3, p.18-23, 1983.

UNDER, P.W.; KASPAR, T. soil compaction and root growth: a review. Agronomy Journal, Madison, v.86, p.759-766, 1994.

VITÓRIA, E.L.; FERNANDES, H.C.; TEIXIERA, M.M.; CECON, P.R.; LACERDA, E.G. Correlação linear e espacial entre produtividade de Brachiaria brizantha, densidade do solo e porosidade total em função do sistema de manejo do solo. Engenharia Agrícola, Jaboticabal, v.32, n.5, p.909-919, 2012. 